\title{
Analysis of Asian Mitochondrial DNA Haplogroups Associated With the Progression of Knee Osteoarthritis in Koreans
}

\author{
Bon San Koo, M.D. ${ }^{1}$, Yoonah Song, M.D. ${ }^{2}$, Seunghun Lee, M.D., Ph.D. ${ }^{2}$, Yoon-Kyoung Sung, M.D., Ph.D. ${ }^{3}$, \\ Kyoung-Jin Shin, M.D., Ph.D. ${ }^{4}$, Nam H. Cho, M.D., Ph.D. ${ }^{5}$, Jae-Bum Jun, M.D., Ph.D. ${ }^{3}$ \\ ${ }^{1}$ Department of Internal Medicine, Inje University Seoul Paik Hospital, Inje University College of Medicine, Departments of ${ }^{2}$ Radiology and \\ ${ }^{3}$ Rheumatology, Hanyang University Hospital for Rheumatic Diseases, ${ }^{4}$ Department of Forensic Medicine, Yonsei University College of \\ Medicine, Seoul, ${ }^{5}$ Department of Preventive Medicine, Ajou University School of Medicine, Suwon, Korea
}

\begin{abstract}
Objective. We investigated Asian mitochondrial DNA (mtDNA) haplogroups associated with knee osteoarthritis (OA) progression in a prospective community-based cohort comprised of Koreans. Methods. Epidemiologic data and Kellgren-Lawrence $(\mathrm{K} / \mathrm{L})$ scores of knee radiographs were obtained from the second (2005 2006) and sixth (2013 2014) follow-up, and patient DNA was analyzed. The mtDNA haplogroup frequencies (M, G, D, D4, D5, M7, M8, M9, M10, N, A, N9, R, F, and B) were compared between the progression (K/L score change on either knee $\geq 2$ or arthroplasty) and non-progression (K/L score change on both knee $\leq 1$ ) groups at the sixth follow-up. Multiple logistic regression was performed to determine relative risk (RRs) of mtDNA haplogroups for OA. Results. In total, 1,115 participants were included, 405 of whom had early OA (higher $\mathrm{K} / \mathrm{L}$ score on both knees of 1 or 2). Among them, 143 and 166 patients were classified in non-progression and progression groups, respectively, at the sixth follow-up. The most frequent haplogroups, B and D4, in Koreans also showed a high frequency in our study. There were no significantly different haplogroups between the non-progression and progression groups. However, the frequency of haplogroup D4 was likely higher in the non-progression group than in the progression group, although not significantly $(13.3 \%$ vs. $7.2 \%, R R=0.51, p=0.081$ in the unadjusted model and $R R=0.56, p=0.149$ in the adjusted model). Conclusion. No significant haplogroups are related to OA progression. Large-scaled studies are needed to reveal the association between mtDNA haplogroups and OA. (J Rheum Dis 2020;27:168-173)
\end{abstract}

Key Words. Korea, Mitochondrial DNA, Osteoarthritis of the knee

\section{INTRODUCTION}

Osteoarthritis $(\mathrm{OA})$ is the most common degenerative disease related to the degradation of articular cartilage. Many studies have been performed to evaluate the relationship between OA and mitochondrial DNA (mtDNA). Changes in intracellular signals such as in mitochondrial respiratory chain activity in chondrocytes may be associated with degenerative changes in cartilage affected by low-grade chronic inflammation [1].

In a previous study, we examined whether mtDNA hap- logroup B contributed to the development of knee OA in Koreans, measured as radiologic changes for approximately 8 years in a large-scale prospective cohort [2]. Identifying the relationship between the haplogroup of mitochondria and development of $\mathrm{OA}$ is important for identifying the risk factors of chronic disease [3-8]. However, considering the nature of OAs, which are characterized by very long-term changes, it is also important to identify factors that can predict which patients with $\mathrm{OA}$ are experiencing rapid OA progression.

Various cohort studies have reported that specific

Received : December 5, 2019, Revised : April 22, 2020, Accepted : May 7, 2020

Corresponding to : Jae-Bum Jun (iDhttp://orcid.org/0000-0002-0208-0505

Department of Rheumatology, Hanyang University Hospital for Rheumatic Diseases, 222-1 Wangsimni-ro, Seongdong-gu, Seoul 04763, Korea. E-mail : junjb@hanyang.ac.kr

Copyright (c) 2020 by The Korean College of Rheumatology.

This is an Open Access article, which permits unrestricted non-commerical use, distribution, and reproduction in any medium, provided the original work is properly cited. 
mtDNA haplogroups are also associated with the radiographic progression of OA [9-11]. They suggested that haplogroups associated with progression differed from the mtDNA haplogroup involved in OA development. Therefore, to determine which mtDNA haplogroups are associated with OA progression in Koreans, we developed a new study design based on a previous study protocol [2].

The aim of this study was to investigate Asian mtDNA haplogroups associated with the progression of knee OA in participants in a prospective ongoing communitybased cohort in Korea.

\section{MATERIALS AND METHODS}

\section{Study design and participants}

As described in our previous study [2], we used the Ansung cohort of an ongoing, prospective cohort study that is part of the Korean Genome and Epidemiology Study [12]. In the present study, mtDNA haplogroups related to the progression of OA were examined by modifying the experimental design of the previous study. Briefly, epidemiologic data and Kellgren-Lawrence $(\mathrm{K} / \mathrm{L})$ scores of the knee radiographs were obtained from the second follow-up (2005 2006) and sixth follow-up (2013 2014) of this cohort. The K/L scores were measured by an orthopedist (KKI) and radiologist (SY) at the second follow-up visit with excellent inter-observer correlation coefficients and by a radiologist (SY) at the sixth follow-up visit with excellent intra-observer correlation coefficients
[13]. The institutional review boards of all involved institutions approved this study (approval no. HYUH 2015-12-022).

Overall, there were 5,018 participants, and we obtained DNA samples from 1,115 participants (Figure 1). We defined early OA as a higher K/L score for both knees of 1 or 2 at the second follow-up to identify progression, rather than as a criterion for the development of OA, as in previous studies of the association between mtDNA and OA development [11]. Among the participants, 405 met the definition of early $\mathrm{OA}$ at the second follow-up and were divided into two groups: $\mathrm{K} / \mathrm{L}$ score change $\leq 1$ in both knees (non-progression group, $\mathrm{n}=143$ ) and $\mathrm{K} / \mathrm{L}$ score change $\geq 2$ in either knee or arthroplasty (progression group, $\mathrm{n}=166$ ) at the sixth follow-up. All missing values for the $\mathrm{K} / \mathrm{L}$ score at the sixth follow-up were excluded $(n=96)$.

\section{mtDNA haplogroup genotyping}

Blood samples were stored in the Korea Biobank Network, Center for Disease Control at the second follow-up visit (2005 2006). Asian mtDNA haplogroups (M, G, D, D4, D5, M7, M8, M9, M10, N, A, N9, R, F, and B) were determined by multiplex mutagenetically separated polymerase chain reaction. Single-nucleotide polymorphisms at positions 15043, 4833, 4883, 3010, 1107, $9824,7196,4491,13152,10873,8794,5417,12705$, and 6392 and a 9-base pair deletion at position 8281 to 8289 were selected and analyzed as described by Lee et al. [14].

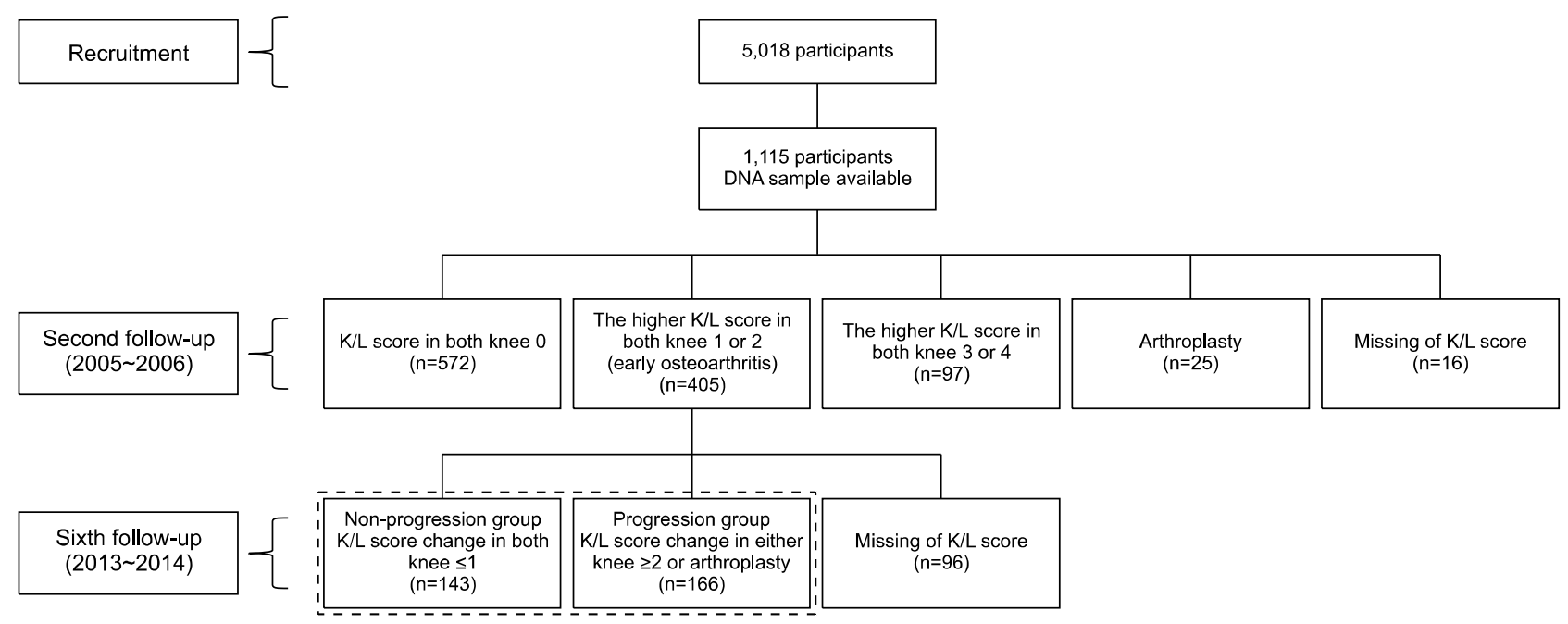

Figure 1. Classification according to the Kellgren-Lawrence $(\mathrm{K} / \mathrm{L})$ score at each follow-up in the study cohort. The frequencies of mitochondrial DNA haplogroups were analyzed in the non-progression and progression groups (box surrounded by the dotted line). 


\section{Statistical analysis}

Differences between the non-progression and progression groups at the sixth follow-up were investigated by Student $t$-test and the Pearson chi-square test. Multiple logistic regression was used to determine the relative risk (RR) of mtDNA haplogroups for OA by adjusting for sex, age, and body mass index (BMI) because the incidence of knee $\mathrm{OA}$ is high in women and the elderly and obesity is a risk factor for OA [15]. Smoking and metabolic syndrome were excluded from the adjusted model because these factors are correlated with sex and BMI, respectively. p-values $<0.05$ indicated statistical significance. All statistical analyses were performed using PASW software version 18.0 (IBM Co., Armonk, NY, USA).

\section{RESULTS}

\section{Baseline clinical characteristics}

The clinical characteristics of the participants are described in Table 1. There were no significant differences in age between the non-progression and progression groups. The number of females was significantly higher in the progression group than in the non-progression group ( $88.6 \%$ vs. $76.9 \%$, respectively). The rates of smoking, drinking, and diabetes, and hypertension were not significantly different between groups. However, the BMI and rates of metabolic syndrome were significantly higher in the progression group than in the non-progression group (26.74 \pm 3.21 vs. $25.33 \pm 3.26$ and $77.7 \%$ vs. $62.0 \%$, respectively).

\section{mtDNA haplogroups associated with non-progression and progression of $\mathrm{OA}$}

The haplogroup frequencies of the non-progression and progression groups and RRs are shown in Table 2. Among the haplogroups, haplogroups B and D4 showed the highest frequencies ( $15.9 \%$ and $10.0 \%$, respectively). In multiple logistic regression analysis, there was no significant $\mathrm{RR}$ for the progression of OA in each haplogroup in the unadjusted model, adjusted model for age, sex, and BMI, and adjusted model for age, sex, BMI, smoking, and metabolic syndrome. Among the haplogroups, the proportion of non-progression patients in haplogroup D4 was likely higher than that in patients showing progression; however, there was also no significant difference between the two groups ( $13.3 \%$ vs. $7.2 \%, \mathrm{RR}=0.51$ [0.24 1.09], $\mathrm{p}=0.081$ in unadjusted model and $\mathrm{RR}=0.56[0.25 \sim$ 1.23], $\mathrm{p}=0.149$ in adjusted model for age, sex, BMI).

\section{DISCUSSION}

Our previous study suggested that participants with haplogroup B had a higher risk of OA development [2]. In the present study, we observed no significant relationship between the haplogroups and OA progression. Haplogroup $\mathrm{B}$, which was associated with the development of OA in our previous study [2], appeared to be related to OA progression but did not show a significant difference in the present study. Haplogroup D4 showed a low frequency in the progression group but the value was not significant.

Several studies in western countries have described the relationship between $\mathrm{OA}$ progression and mtDNA haplogroups. Soto-Hermida et al. [10] found that patients with haplogroup $\mathrm{T}$ had the lowest increase in $\mathrm{K} / \mathrm{L}$ score (hazard ratio $=0.499 ; 95 \%$ confidence interval [95\% CI]: $0.261 \sim 0.815$ ) and in other radiographic indicators for progression such as joint space narrowing, osteophytes, and subchondral sclerosis. They also studied OA progression and mtDNA haplogroups in a Spanish cohort [11]. Patients in cluster TJ showed slower radio-

Table 1. Clinical characteristics of participants included in the cohort

\begin{tabular}{lcrrr}
\hline \multicolumn{1}{c}{ Variable } & Non-progression $(\mathrm{n}=143)$ & Progression $(\mathrm{n}=166)$ & $\mathrm{p}$-value & $\mathrm{p}$-value* \\
\hline Age $(\mathrm{yr})$ & $62.47 \pm 8.22$ & $62.88 \pm 6.17$ & 0.624 & - \\
Sex, female & $110(76.9)$ & $147(88.6)$ & 0.006 & 0.007 \\
Body mass index $\left(\mathrm{kg} / \mathrm{m}^{2}\right)$ & $25.33 \pm 3.26$ & $26.74 \pm 3.21$ & $<0.001$ & $<0.001$ \\
Smoking, ever/current & $26(18.2)$ & $19(11.4)$ & 0.094 & 0.087 \\
Drinking, ever/current & $53(37.1)$ & $48(28.9)$ & 0.128 & 0.134 \\
Diabetes & $34(24.3)$ & $31(18.9)$ & 0.254 & 0.234 \\
Hypertension & $70(49.0)$ & $95(57.2)$ & 0.146 & 0.162 \\
Metabolic syndrome & $88(62.0)$ & $129(77.7)$ & 0.003 & 0.003 \\
\hline
\end{tabular}

Values are presented as mean \pm standard deviation or number (\%). *Age-adjusted. 
Table 2. Multiple logistic regression analysis of mitochondrial haplogroups associated with osteoarthritis

\begin{tabular}{|c|c|c|c|c|c|c|c|c|}
\hline \multirow{2}{*}{ Haplogroup } & \multirow{2}{*}{$\begin{array}{c}\text { Non- } \\
\text { progression } \\
(n=143)\end{array}$} & \multirow{2}{*}{$\begin{array}{l}\text { Progression } \\
\quad(n=166)\end{array}$} & \multicolumn{3}{|c|}{ Unadjusted } & \multicolumn{3}{|c|}{ Adjusted* } \\
\hline & & & B & $\mathrm{RR}(95 \% \mathrm{Cl})$ & p-value & B & $\mathrm{RR}(95 \% \mathrm{Cl})$ & $\mathrm{p}$-value \\
\hline A & $14(9.8)$ & $16(9.6)$ & -0.017 & $0.98(0.46 \sim 2.09)$ & 0.964 & -0.167 & $0.85(0.38 \sim 1.87)$ & 0.680 \\
\hline B & $21(14.7)$ & $28(16.9)$ & 0.164 & $1.18(0.64 \sim 2.18)$ & 0.601 & 0.185 & $1.20(0.63 \sim 2.28)$ & 0.571 \\
\hline $\mathrm{D}$ & $1(0.7)$ & $0(0.0)$ & -21.359 & - & 1.000 & -20.721 & - & 1.000 \\
\hline D4 & $19(13.3)$ & $12(7.2)$ & -0.676 & $0.51(0.24 \sim 1.09)$ & 0.081 & -0.586 & $0.56(0.25 \sim 1.23)$ & 0.149 \\
\hline D4a & $9(6.3)$ & $9(5.4)$ & -0.158 & $0.85(0.33 \sim 2.21)$ & 0.744 & -0.232 & $0.79(0.30 \sim 2.12)$ & 0.644 \\
\hline $\mathrm{D} 4 \mathrm{~b}$ & $2(1.4)$ & $1(0.6)$ & -0.850 & $0.43(0.04 \sim 4.76)$ & 0.489 & -0.637 & $0.53(0.05 \sim 6.06)$ & 0.608 \\
\hline D4b2 & $9(6.3)$ & $11(6.6)$ & 0.055 & $1.06(0.42 \sim 2.63)$ & 0.906 & 0.112 & $1.12(0.44 \sim 2.87)$ & 0.816 \\
\hline D4j & $3(2.1)$ & $2(1.2)$ & -0.564 & $0.57(0.09 \sim 3.45)$ & 0.540 & 0.017 & $1.02(0.15 \sim 6.77)$ & 0.986 \\
\hline $\mathrm{D} 4 \mathrm{e}$ & $4(2.8)$ & $7(4.2)$ & 0.425 & $1.53(0.44 \sim 5.34)$ & 0.505 & 0.401 & $1.49(0.41 \sim 5.39)$ & 0.540 \\
\hline $\mathrm{D} 4 \mathrm{a} / \mathrm{D} 4 \mathrm{~b}$ & $0(0.0)$ & $1(0.6)$ & 21.060 & - & 1.000 & 20.804 & - & 1.000 \\
\hline D5 & $12(8.4)$ & $13(7.8)$ & -0.075 & $0.93(0.41 \sim 2.10)$ & 0.857 & 0.003 & $1.00(0.43 \sim 2.34)$ & 0.994 \\
\hline $\mathrm{F}$ & $11(7.7)$ & $17(10.2)$ & 0.314 & $1.37(0.62 \sim 3.03)$ & 0.438 & 0.277 & $1.32(0.58 \sim 2.99)$ & 0.508 \\
\hline G & $8(5.6)$ & $11(6.6)$ & 0.180 & $1.20(0.47 \sim 3.06)$ & 0.707 & 0.184 & $1.20(0.46 \sim 3.16)$ & 0.709 \\
\hline M & $1(0.7)$ & $2(1.2)$ & 0.549 & $1.73(0.16 \sim 19.30)$ & 0.655 & 0.488 & $1.63(0.14 \sim 18.47)$ & 0.694 \\
\hline M7 & $9(6.3)$ & $12(7.2)$ & 0.149 & $1.16(0.47 \sim 2.84)$ & 0.745 & 0.049 & $1.05(0.42 \sim 2.62)$ & 0.916 \\
\hline M8 & $8(5.6)$ & $11(6.6)$ & 0.180 & $1.20(0.47 \sim 3.06)$ & 0.707 & -0.010 & $0.99(0.37 \sim 2.63)$ & 0.984 \\
\hline M9 & $3(2.1)$ & $2(1.2)$ & -0.564 & $0.57(0.09 \sim 3.45)$ & 0.540 & -0.384 & $0.68(0.11 \sim 4.26)$ & 0.681 \\
\hline M10 & $2(1.4)$ & $0(0.0)$ & -21.366 & - & 0.999 & -22.129 & - & 0.999 \\
\hline N9 & $7(4.9)$ & $11(6.6)$ & 0.321 & $1.38(0.52 \sim 3.66)$ & 0.519 & 0.398 & $1.49(0.54 \sim 4.10)$ & 0.440 \\
\hline $\mathrm{R}$ & $0(0.0)$ & $0(0.0)$ & - & - & - & - & - & - \\
\hline $\begin{array}{l}\text { Macro- } \\
\text { haplogroup } \mathrm{M}^{\dagger}\end{array}$ & $90(62.9)$ & $94(56.6)$ & -0.263 & $0.77(0.49 \sim 1.21)$ & 0.260 & -0.227 & $0.80(0.50 \sim 1.28)$ & 0.348 \\
\hline $\begin{array}{l}\text { Macro- } \\
\text { haplogroup } \mathrm{N}^{\ddagger}\end{array}$ & $53(37.1)$ & $72(43.4)$ & 0.263 & $1.30(0.82 \sim 2.06)$ & 0.260 & 0.227 & $1.26(0.78 \sim 2.02)$ & 0.348 \\
\hline $\begin{array}{l}\text { Macro- } \\
\text { haplogroup } \mathrm{D}^{\S}\end{array}$ & $59(41.3)$ & $56(33.7)$ & -0.322 & $0.72(0.46 \sim 1.15)$ & 0.173 & -0.209 & $0.81(0.50 \sim 1.31)$ & 0.394 \\
\hline $\begin{array}{l}\text { Macro- } \\
\text { haplogroup R\| }\end{array}$ & $32(22.4)$ & $45(27.1)$ & 0.255 & $1.29(0.77 \sim 2.17)$ & 0.338 & 0.255 & $1.29(0.75 \sim 2.22)$ & 0.357 \\
\hline
\end{tabular}

Values are presented as number (\%). RR: relative risk, $\mathrm{Cl}$ : confidence interval. *Adjusted for age, sex, body mass index. ${ }^{\dagger}$ Macro-haplogroupM: D, D4, D4a, D4b, D4b2, D4j, D4e, D4a/D4b, D5, G, M, M7, M8, M9, and M10. ${ }^{\ddagger}$ Macro-haplogroup N: A, B, F, N9, and R. ${ }^{\S}$ Macro-haplogroup D: D, D4, D4a, D4b, D4b2, D4j, D4e, D4a/D4b, and D5. "Macro-haplogroup R: B, F, and R.

graphic OA progression than patients in cluster $\mathrm{KU}$ (hazard ratio $=1.711 ; 95 \% \mathrm{CI}: 1.037 \sim 2.823$ ).

In a case-control study of Asians, Fang et al. [16] reported that haplogroup $\mathrm{G}$ increased the risk of OA occurrence (OA group $4.3 \%$ vs. control $1.4 \%$, odds ratio $[\mathrm{OR}]=3.834 ; \mathrm{p}=0.03$ ) and patients with haplogroup $\mathrm{G}$ showed a higher severity of progression (K/L score 4$)$ of knee OA $(\mathrm{OR}=10.870, \mathrm{p}=0.007)$. Additionally, they showed that haplogroup D4/D4a was related to the higher-severity OA. Although the designs of their studies differed from those of our cohort study, the frequency of haplogroup D4 may be lower in the progression group than in the non-progression group. In East Asians, the frequent sequence variations in the Korean population were very similar to those in Japanese and Northern
Chinese populations [17]. However, the frequency of the haplogroups related to disease may vary by country.

In the previous study investigating the progression of $\mathrm{OA}$, a $\mathrm{K} / \mathrm{L}$ score change $\geq 1$ was defined as OA progression $[10,11]$. However, we defined $\mathrm{K} / \mathrm{L}$ score change $>2$ as a progression after approximately 8 years among patients who had a higher K/L score in both knees of 1 or 2 at the second (baseline) follow-up. Even when using this strict definition of OA progression, no meaningful haplogroup was identified. This result is thought to be related to the small number of participants defined as having early OA. However, this is the only study in Korea to evaluate the association between knee OA progression and mtDNA haplogroups. Additional large-scale studies are necessary to identify the mtDNA haplogroup related 
to OA, which will improve the early diagnosis and prevention in patients at a high risk of OA.

Several studies have suggested that haplogroup D4 is related to type 2 diabetes mellitus (DM). Liou et al. [18] suggested that haplogroup B4 was significantly associated with DM (OR 1.54 [95\% CI 1.18 2.02], $\mathrm{p}<$ 0.001), whereas haplogroup D4 showed borderline resistance against type 2 DM (OR 0.68 [95\% CI 0.49 0.94], $\mathrm{p}=0.02$ ) in a Chinese population in Taiwan [18]. However, Jiang et al. [19] suggested that haplogroup D4 is associated with an increased risk of developing type 2 DM (OR 1.47 [95\% CI 1.22 1.77], $\mathrm{p}<0.01$ ) in a Uyghur population in China and that the $3010 \mathrm{G}>\mathrm{A}$ variant is likely involved in the pathogenesis of type $2 \mathrm{DM}$. Fuku et al. [20] also suggested that haplogroup D4b in Korean men was associated with an increased risk of DM (OR 3.55 [95\% CI 1.65 8.34], $\mathrm{p}<0.01$ ). Although the relationship between DM and haplogroup D4 shows variable results, considering that DM is associated with OA [21], haplogroup D4 may be associated with OA in Koreans.

Our study had several limitations. First, we investigated the progression of knee $\mathrm{OA}$ according to the $\mathrm{K} / \mathrm{L}$ score change in knee radiographs after approximately 8 years. This follow-up period may not be sufficient to observe the progression of OA on knee radiographs. Long-term research designs using elaborate degenerative change screening methods are required to acquire more participants. Second, knee OA is generally defined as a $\mathrm{K} / \mathrm{L}$ score of 2 or more; however, in our study, participants with a $\mathrm{K} / \mathrm{L}$ score $\geq 1$ at baseline were defined as having $\mathrm{OA}$, as described previously [11]. Although the definition we used led to a larger number of participants, the sample size was still too small to obtain meaningful results. Third, although the parameters were adjusted for OA progression, we also need to consider variables related to risk factors for OA, such as anatomic factors, bone density, and physical activity. Fourth, functional scores such as the Western Ontario and McMaster Universities (WOMAC) is important for evaluating dysfunction in patients with OA. However, this information was not available for the Ansung cohort. The WOMAC score can complement the definition of progression by the $\mathrm{K} / \mathrm{L}$ score.

\section{CONCLUSION}

In conclusion, no mtDNA haplogroup was found to be associated with the progression of $\mathrm{OA}$ in Koreans. Although not significant, haplogroup D4 may be asso- ciated with slower progression of OA. Large-scale studies are needed to determine the relationship between mtDNA haplogroups and OA.

\section{ACKNOWLEDGMENTS}

This research was supported by the Basic Science Research Program through the National Research Foundation of Korea (NRF) funded by the Ministry of Education (NRF-2015R1D1A1A01057559).

\section{CONFLICT OF INTEREST}

No potential conflict of interest relevant to this article was reported.

\section{AUTHOR CONTRIBUTIONS}

B.S.K, Y.K.S, and J.B.J. were involved in conception and design, interpretation of data. K.J.S. and N.H.C were involved in acquisition and analysis of data. Y.S., S.L., K.J.S., N.H.C., and J.B.J. were involved in acquisition and interpretation of data. All authors were involved in drafting and revising the manuscript critically for important intellectual content and final approval of the version to be published.

\section{REFERENCES}

1. Blanco FJ, Valdes AM, Rego-Pérez I. Mitochondrial DNA variation and the pathogenesis of osteoarthritis phenotypes. Nat Rev Rheumatol 2018;14:327-40.

2. Koo BS, Song Y, Lee S, Sung YK, Shin KJ, Cho NH, et al. Association of Asian mitochondrial DNA haplogroup B with new development of knee osteoarthritis in Koreans. Int J Rheum Dis 2019;22:411-6.

3. Blanco FJ, Rego I, Ruiz-Romero C. The role of mitochondria in osteoarthritis. Nat Rev Rheumatol 2011;7:161-9.

4. Fernández-Moreno $\mathrm{M}$, Soto-Hermida $\mathrm{A}$, Vázquez-Mosquera ME, Cortés-Pereira E, Relaño S, Hermida-Gómez T, et al. Mitochondrial DNA haplogroups influence the risk of incident knee osteoarthritis in OAI and CHECK cohorts. A meta-analysis and functional study. Ann Rheum Dis 2017; 76:1114-22.

5. Lotz M, Martel-Pelletier J, Christiansen C, Brandi ML, Bruyère $\mathrm{O}$, Chapurlat $\mathrm{R}$, et al. Value of biomarkers in osteoarthritis: current status and perspectives. Ann Rheum Dis 2013;72:1756-63.

6. Minafra L, Bravatà V, Saporito M, Cammarata FP, Forte GI, Caldarella S, et al. Genetic, clinical and radiographic signs in knee osteoarthritis susceptibility. Arthritis Res Ther 2014;16:R91.

7. Rego I, Fernández-Moreno M, Fernández-López C, Gómez- 
Reino JJ, González A, Arenas J, et al. Role of European mitochondrial DNA haplogroups in the prevalence of hip osteoarthritis in Galicia, Northern Spain. Ann Rheum Dis 2010;69:210-3.

8. Rego-Pérez I, Fernández-Moreno M, Fernández-López C, Arenas J, Blanco FJ. Mitochondrial DNA haplogroups: role in the prevalence and severity of knee osteoarthritis. Arthritis Rheum 2008;58:2387-96.

9. Fernández-Moreno M, Soto-Hermida A, Vázquez-Mosquera ME, Cortés-Pereira E, Pértega S, Relaño S, et al. A replication study and meta-analysis of mitochondrial DNA variants in the radiographic progression of knee osteoarthritis. Rheumatology (Oxford) 2017;56:263-70.

10. Soto-Hermida A, Fernández-Moreno M, Oreiro N, FernándezLópez C, Pértega S, Cortés-Pereira E, et al. Mitochondrial DNA (mtDNA) haplogroups influence the progression of knee osteoarthritis. Data from the Osteoarthritis Initiative (OAI). PLoS One 2014;9:e112735.

11. Soto-Hermida A, Fernández-Moreno M, Pértega-Díaz S, Oreiro N, Fernández-López C, Blanco FJ, et al. Mitochondrial DNA haplogroups modulate the radiographic progression of Spanish patients with osteoarthritis. Rheumatol Int 2015;35:337-44

12. Cho NH, Kim JY, Kim SS, Shin C. The relationship of metabolic syndrome and constitutional medicine for the prediction of cardiovascular disease. Diabetes Metab Syndr 2013;7:226-32.

13. Cho NH, Song Y, Lee S, Sung YK, Jun JB. Incidence of knee chondrocalcinosis and its risk factors in a community-based cohort. Int J Rheum Dis 2018;21:1391-7.

14. Lee HY, Yoon JA, Yang WI, Shin KJ. A one step multiplex
PCR assay for rapid screening of East Asian mtDNA haplogroups on forensic samples. Leg Med (Tokyo) 2013;15: 50-4.

15. Allen KD, Golightly YM. State of the evidence. Curr Opin Rheumatol 2015;27:276-83.

16. Fang $\mathrm{H}$, Zhang F, Li F, Shi $\mathrm{H}$, Ma L, Du M, et al. Mitochondrial DNA haplogroups modify the risk of osteoarthritis by altering mitochondrial function and intracellular mitochondrial signals. Biochim Biophys Acta 2016;1862: 829-36.

17. Park S, Cho S, Seo HJ, Lee JH, Kim MY, Lee SD. Entire mitochondrial DNA sequencing on massively parallel sequencing for the Korean population. J Korean Med Sci 2017;32:587-92.

18. Liou CW, Chen JB, Tiao MM, Weng SW, Huang TL, Chuang $\mathrm{JH}$, et al. Mitochondrial DNA coding and control region variants as genetic risk factors for type 2 diabetes. Diabetes 2012;61:2642-51.

19. Jiang W, Li R, Zhang Y, Wang P, Wu T, Lin J, et al. Mitochondrial DNA mutations associated with type 2 diabetes mellitus in Chinese uyghur population. Sci Rep 2017;7:16989.

20. Fuku N, Park KS, Yamada Y, Nishigaki Y, Cho YM, Matsuo $\mathrm{H}$, et al. Mitochondrial haplogroup N9a confers resistance against type 2 diabetes in Asians. Am J Hum Genet 2007; 80:407-15

21. Williams MF, London DA, Husni EM, Navaneethan S, Kashyap SR. Type 2 diabetes and osteoarthritis: a systematic review and meta-analysis. J Diabetes Complications 2016;30:944-50. 